\title{
ON THE SPECTRAL SYNTHESIS OF BOUNDED FUNCTIONS
}

\section{MASAKITI KINUKAWA}

0 . In this note, we intend to generalize a theorem on the spectral synthesis of bounded functions due to A. Beurling [2] and to discuss an analogous problem in the case of bounded sequences. For all terms not explained here, the reader is referred to the papers of $A$. Beurling [2] and J. P. Kahane [4].

1. For a function $\phi(x) \in L^{\infty}(-\infty, \infty)$, we shall denote its spectral set by $\operatorname{Sp} .(\phi)$. We shall be concerned with the space $\mathfrak{A}$ of Fourier transforms of functions in $L^{1}(-\infty, \infty)$. That is, $f(t) \in \mathfrak{A}$ means that there exists a function $F(x) \in L^{1}(-\infty, \infty)$ whose Fourier transform is $f(t)$. We introduce a norm $\|f\|_{\mathfrak{A}}$ in the space $\mathfrak{A}$ defining it by

$$
\|f\|_{\mathfrak{A}}=\int_{-\infty}^{\infty}|F(x)| d x
$$

We say $g(t)=T(f(t))$ (or $T$ ) is a normalized contraction of $f$ when the complex function $T(z)$ satisfies the Lipschitz condition $\left|T(z)-T\left(z^{\prime}\right)\right|$ $\leqq\left|z-z^{\prime}\right|$ and $\lim _{t \rightarrow \infty} g(t)=0$. Moreover, we say an element $f$ of $\mathfrak{A}$ is contractible in the space $\mathfrak{A}$ if every normalized contraction of $f$ also belongs to the space $\mathfrak{A}$. And we say $f$ is uniformly contractible in the space $\mathfrak{A}$ if $f$ is contractible in $\mathfrak{A}$ and if $\lim _{n \rightarrow \infty}\left\|g_{n}\right\| \mathfrak{A}=0$ for any sequence $\left\{g_{n}(t)\right\}$ of normalized contractions of $f(t)$ such that $\lim _{n \rightarrow \infty} g_{n}(t)=0$.

Some of Beurling's theorems [2] may be read as follows:

Theorem 1. Suppose that

(i) $f(t)$ is uniformly contractible in the space $\mathfrak{A}$, and

(ii) $f(t)=\int_{-\infty}^{\infty} e^{i x t} F(x) d x$ vanishes on $\mathrm{Sp} .(\phi)$.

Then we have

(iii) $\int_{-\infty}^{\infty} \phi(x) F(x) d x=0$.

Theorem 2. Suppose that

(iv) $w(x) \in L^{1}(-\infty, \infty)$ and $w(x)$ is even, positive and nonincreasing in $(0, \infty)$, and

(v) $|F(x)| \leqq w(|x|)$.

Then the Fourier transform $f$ of $F$ is uniformly contractible in the space $\mathfrak{A}$.

Note that the assumptions (ii), (iv) and (v) imply the conclusion (iii). This means that the spectral synthesis of a bounded function $\phi$

Received by the editors April 4, 1962. 
is possible with respect to the topology defined by the norm $\|\psi\|_{\infty, w}$ $=\int_{-\infty}^{\infty}|\psi(x)| w(x) d x$ for $\psi \in L^{\infty}(-\infty, \infty)$.

The first purpose of this note is to generalize the assumption (iv). In fact, we shall prove the following:

THEOREM 3. The assumption (iv) in Theorem 2 can be replaced by the following conditions:

(iv) ${ }^{*} w(x)$ is even, positive and satisfies

$$
\int_{0}^{\infty} x^{-3 / 2}\left(\int_{0}^{x} u^{2} w^{2}(u) d u\right)^{1 / 2} d x+\int_{0}^{\infty} x^{-1 / 2}\left(\int_{x}^{\infty} w^{2}(u) d u\right)^{1 / 2} d x<\infty .
$$

Proof of Theorem 3. In a previous paper (M. Kinukawa [5]), we proved that $f$ is contractible in $\mathfrak{A}$ under the assumptions of Theorem 3. That is, for any normalized contraction $g(t)$ of $f(t)$, there exists a function $G(x) \in L^{1}(-\infty, \infty)$ such that $g(t)$ is the Fourier transform of $G(x)$. Let $\left\{g_{n}(t)\right\}$ be a sequence of contractions of $f(t)$ such that $\lim _{n \rightarrow \infty} g_{n}(t)=0$. Then, we only need for our purpose to show that

$$
\lim _{n \rightarrow \infty}\left\|g_{n}\right\|_{\mathfrak{A}}=\lim _{n \rightarrow \infty} \int_{-\infty}^{\infty}\left|G_{n}(x)\right| d x=0,
$$

where each $G_{n}(x)$ is a function whose Fourier transform is $g_{n}(t)$. (This means that $f(t)$ is uniformly contractible in the space $\mathfrak{A}$.) For this purpose, we need the following inequality which was suggested by Professors S. and M. Izumi:

$$
\int_{0}^{\infty}|G(x)| d x \leqq \int_{0}^{\infty} x^{-3 / 2}\left(\int_{0}^{x} u^{2}|G(u)|^{2} d u\right)^{1 / 2} d x
$$

To prove the above inequality, we may suppose that $G(x)=0$ for $x \geqq N>0$. Putting $S(x)=\int_{0}^{x} u|G(u)| d u$, we have

$$
\begin{aligned}
\int_{0}^{\infty}|G(x)| d x & =\int_{0}^{N}|G(x)| d x=\int_{0}^{N} x^{-1} \frac{d}{d x} S(x) d x \\
& =\int_{0}^{N} u^{-2} S(u) d u+N^{-1} S(N) .
\end{aligned}
$$

Since, by Schwarz's inequality, $S(x) \leqq x^{1 / 2}\left(\int_{0}^{x} u^{2}|G(u)|^{2} d u\right)^{1 / 2}$, we get

$$
\begin{aligned}
\int_{0}^{\infty}|G(x)| d x \leqq & \int_{0}^{N} x^{-3 / 2}\left(\int_{0}^{x} u^{2}|G(u)|^{2} d u\right)^{1 / 2} d x \\
& +2 N^{-1 / 2}\left(\int_{0}^{N} u^{2}|G(u)|^{2} d u\right)^{1 / 2}
\end{aligned}
$$


where the second part in the right-hand side is

$$
\int_{N}^{\infty} x^{-3 / 2} d x\left(\int_{0}^{N} u^{2}|G(u)|^{2} d u\right)^{1 / 2} \leqq \int_{N}^{\infty} x^{-3 / 2}\left(\int_{0}^{x} u^{2}|G(u)|^{2} d u\right)^{1 / 2} d x
$$

Thus we get the inequality (1.2).

We now proceed to prove (1.1). We have

$$
\begin{aligned}
\int_{0}^{x} u^{2}\left|G_{n}(u)\right|^{2} d u & \leqq C x^{2} \int_{0}^{x}\left|G_{n}(u)\right|^{2} \sin ^{2} \frac{u}{x} d u \\
& \leqq C x^{2} \int_{-\infty}^{\infty}\left|G_{n}(u)\right|^{2} \sin ^{2} \frac{u}{x} d u
\end{aligned}
$$

Then, by Parseval's relation, the right-hand side is equal to

$$
\begin{aligned}
& =C x^{2} \int_{-\infty}^{\infty}\left|g_{n}(u+1 / x)-g_{n}(u-1 / x)\right|^{2} d u \\
& \leqq C x^{2} \int_{-\infty}^{\infty}|f(u+1 / x)-f(u-1 / x)|^{2} d u \\
& =C x^{2} \int_{-\infty}^{\infty}|F(u)|^{2} \sin ^{2} \frac{u}{x} d u \\
& \leqq C x^{2} \int_{0}^{\infty} w^{2}(u) \sin ^{2} \frac{u}{x} d u \\
& \leqq C\left\{\int_{0}^{x} u^{2} w^{2}(u) d u+x^{2} \int_{x}^{\infty} v^{2}(u) d u\right\} .
\end{aligned}
$$

From the inequalities (1.2), (1.3), (1.4) and (1.5), we get

$$
\begin{aligned}
\left\|g_{n}\right\|_{\mathfrak{x}} \leqq & C \int_{-\infty}^{\infty} d x\left\{|x|^{-1} \int_{-\infty}^{\infty}\left|g_{n}(u+1 / x)-g_{n}(u-1 / x)\right|^{2} d u\right\}^{1 / 2} \\
\leqq & C \int_{-\infty}^{\infty} d x\left\{|x|^{-1} \int_{-\infty}^{\infty}|f(u+1 / x)-f(u-1 / x)|^{2} d u\right\}^{1 / 2} \\
\leqq & C \int_{0}^{\infty} x^{-3 / 2}\left(\int_{0}^{x} u^{2} w^{2}(u) d u\right)^{1 / 2} d x \\
& +C \int_{0}^{\infty} x^{-1 / 2}\left(\int_{x}^{\infty} w^{2}(u) d u\right)^{1 / 2} d x \\
< &
\end{aligned}
$$

Note that $\left|g_{n}(u+1 / x)-g_{n}(u-1 / x)\right| \leqq|f(u+1 / x)-f(u-1 / x)|$; then, 
by the above inequality and by Lebesgue's convergence theorem, we see that

$$
\lim _{n \rightarrow \infty}\left\|g_{n}\right\|_{\mathfrak{A}}=0
$$

which completes the proof of Theorem 3.

2. We can discuss the spectral synthesis problem of bounded sequences analogously to the case in $\$ 1$. In the case of bounded sequences, the space of absolutely convergent Fourier series, which we shall denote by $A$, plays the role of the space $\mathfrak{A}$ in the preceding section.

Suppose that $f(x)=\sum_{n=-\infty}^{\infty} c_{n} e^{i n x}$ belongs to the space $A$, and define a norm of $f(x)$ in $A$ by $\|f\|_{A}=\sum_{n=-\infty}^{\infty}\left|c_{n}\right|$. Let us suppose that a transformation $T(z)$ satisfies $T(0)=0$ and $\left|T(z)-T\left(z^{\prime}\right)\right| \leqq\left|z-z^{\prime}\right|$; then we can define the terms "contractible" and "uniformly contractible" in the space $A$ in a way similar to that used for the space $\mathfrak{A}$. (In this case, we omit the word "normalized" from the corresponding definitions.) Let a sequence $\left\{\phi_{n}\right\}$ be bounded and denote its spectral set by Sp. $\left(\phi_{n}\right)$. Then the theorems corresponding to Theorems 1 and 3 can be stated in the following way:

TheOREm 4. Suppose that $f(x) \in A$ is uniformly contractible in the space $A$ and $f(x)=\sum_{n=-\infty}^{\infty} c_{n} e^{i n x}$ vanishes on $\operatorname{Sp} .\left(\phi_{n}\right)$. Then we have $\sum_{n=-\infty}^{\infty} \phi_{n} c_{n}=0$.

THEOREM 5. Suppose that $\left\{w_{n}\right\}$ is a positive sequence such that $w_{-n}=w_{n}$ and satisfies the following condition

$$
\sum_{n=1}^{\infty} n^{-3 / 2}\left(\sum_{k=1}^{n} k^{2} w_{k}^{2}\right)^{1 / 2}+\sum_{n=1}^{\infty} n^{-1 / 2}\left(\sum_{k=n+1}^{\infty} w_{k}^{2}\right)^{1 / 2}<\infty .
$$

Then an element $f(x)$ of $A$ is uniformly contractible in the space $A$ when the Fourier coefficients $\left\{c_{n}\right\}$ of $f$ satisfy $\left|c_{n}\right| \leqq w_{|n|}$.

\section{REFERENCES}

1. A. Beurling, Sur une classe de fonctions presque-périodiques, C. R. Acad. Sci. Paris 225 (1947), 326-327.

2. - On the spectral synthesis of bounded functions, Acta Math. 81 (1949), 225-238.

3. R. P. Boas, Beurling's test for absolute convergence of Fourier series, Bull. Amer. Math. Soc. 66 (1960), 24-26.

4. J. P. Kahane, Sur la synthèse harmonique dans $1^{\infty}$, Acad. Brasil. Ciencias 32 (1960), 179-189.

5. M. Kinukawa, Contractions of Fourier coefficients and Fourier integrals, J. Analyse Math. 8 (1960-61), 377-406.

Japan International Christian University, Tokyo 